\title{
Management of anemia in inflammatory bowel disease: a challenge in everyday clinical practice
}

\author{
Edyta Tulewicz-Marti ${ }^{1}$, Andrzej Moniuszko ${ }^{1}$, Grażyna Rydzewska ${ }^{1,2}$ \\ ${ }^{1}$ Department of General Medicine and Gastroenterology with Inflammatory Bowel Disease Subdivision, Central Clinical \\ Hospital of the Ministry of Interior Affairs and Administration, Warsaw, Poland \\ ${ }^{2}$ Faculty of Medicine and Health Sciences, Jan Kochanowski University, Kielce, Poland
}

Gastroenterology Rev 2017; 12 (4): 239-243

DOI: https://doi.org/10.5114/pg.2017.72096

Key words: anemia, inflammatory bowel disease, hypophosphatemia, FGF23.

Address for correspondence: Edyta Tulewicz-Marti MD, Department of General Medicine and Gastroenterology with Inflammatory Bowel Disease Subdivision, Central Clinical Hospital of the Ministry of Interior Affairs and Administration, 137 Woloska St, 02-507 Warsaw, Poland, phone: +48 22508 12 40, fax: +48 22508 10 40, e-mail: e.tulewicz@gmail.com

\begin{abstract}
Anemia is the most common extraintestinal manifestation and complication of inflammatory bowel disease (IBD). Its etiology is multifactorial and mostly is a combination of iron deficiency anemia (IDA) and chronic anemia (ACD). Because of its high incidence in patients with IBD and its influence on their quality of life, regular screening is recommended. In case of IDA type of medication and route of administration should be determined by many factors such as general condition of the patient, IBD activity or anemia severity. Intravenous iron supplementation is the preferred route but may be associated with phosphate drop or even severe hypophosphatemia (HP). The mechanism of HP related to the intravenous iron infusions is not clearly known yet, but it might be related to the change of FGF-23 levels. What more not all parenteral forms of iron are equal and some may have a higher risk of HP than others.
\end{abstract}

\section{Introduction}

Inflammatory bowel disease (IBD), encompassing Crohn's disease (CD) and ulcerative colitis (CU), is a chronic gastrointestinal disease characterized by a history of exacerbations and remissions and a multifactorial, not fully understood etiology. It is probably influenced by a genetic predisposition, the enteric microflora and environmental factors. The most common symptoms of IBD are related to chronic inflammation of the digestive system such as diarrhea and abdominal pain. They may be accompanied by different extraintestinal manifestations which involve the musculoskeletal (e.g. arthritis), dermatologic (e.g. erythema nodosum or pyoderma gangrenosum), and hepatopancreatobiliary system (e.g. primary sclerosing cholangitis) or ocular lesions (e.g. uveitis). The most common extraintestinal manifestation and complication of IBD is anemia [1], which affects up to $74 \%$ of patients [2, 3]. According to the ECCO-EpiCom study it affects approximately $42 \%$ during the first year from diagnosis [4]. In most cases, it is a combination of iron deficiency anemia (IDA) and chronic anemia (ACD). Other causes include vitamin $B_{12}$ and folate deficiency or the toxic effect of medications. Anemia is not just a laboratory parameter but is a serious medical condition significantly affecting the quality of life [5, 6] and work of IBD patients [7], which also increases hospitalizations and generates additional health care costs.

\section{Diagnosis and prevalence of anemia in inflammatory bowel disease}

According to the 1968 definition by the World Health Organization, still used today, anemia is a condition in which the number of red blood cells or their oxygen-carrying capacity is insufficient to meet physiologic needs, which vary by age, sex, altitude, smoking, and pregnancy status [8]. The overall anemia cut-offs, presented in Table I, also apply to IBD patients and should be an aim of the iron supplementation. African Americans have lower limits of hemoglobin $-11.5 \mathrm{~g} / \mathrm{dl}$ for women and $12.9 \mathrm{~g} / \mathrm{dl}$ for men.

At the time of diagnosis, about $40 \%$ of adult patients have anemia. In the pediatric population these 
Table I. Hemoglobin levels to diagnose anemia at sea level

\begin{tabular}{lcc} 
Population & $\mathrm{Hg}[\mathrm{g} / \mathrm{dl}]$ & $\mathrm{Ht}(\%)$ \\
\hline Children 6-59 months of age & 11 & 33 \\
\hline Children 5-11 years of age & 11.5 & 34 \\
\hline Children > 12 years of age & 12 & 36 \\
\hline Pregnant women & 11 & 33 \\
\hline Non-pregnant women & 12 & 36 \\
\hline Men (15 years and above) & 13 & 39
\end{tabular}

figures are higher and may be $75 \%[9,10]$, while iron deficiency can be up to 85\% [11]. In the Swedish IBSEN study (a 10-year follow-up of more than 700 patients with (BD), anemia was more common in patients with $C D$ than $C U$ and was more common in women with $C D$ after 10 years of treatment [12].

Because of the high incidence of anemia in patients with IBD and its influence on quality of life, regular screening of patients is recommended. In accordance with ECCO recommendations (1B) [13] and recommendations of the Polish National Consultant in Gastroenterology, regular follow-ups of patients in remission should be performed every 6-12 months, and of patients with active disease every 3 months [14, 15]. For laboratory screening, full blood count, C-reactive protein (CRP), and serum ferritin should be used. In patients at risk of vita$\min B_{12}$ and folate deficiency or in case of macrocytosis in absence of therapy with thiopurines, the level of $B_{12}$ and folate should be measured at least annually.

\section{Anemia work-up}

The initial work-up of anemia should start with evaluation of mean corpuscular volume (MCV), helping to distinguish between different etiologies: microcytosis such as IDA, macrocytosis (vitamin $B_{12}$ and folate deficiency or thiopurine treatment) or normocytosis (ACD). Secondly, reticulocyte count should be evaluated in order to assess the ability of bone marrow to respond properly to anemia. Low or normal reticulocyte count may indicate inappropriate erythropoiesis or primary bone marrow disease, while increased reticulocytes indicate correct red cell formation. Moreover, normal platelet and white blood cells count exclude pancytopenia. Incorrect haptoglobin, lactate dehydrogenase and bilirubin levels may indicate hemolysis.

\section{Iron deficiency anemia}

Iron is an essential substrate in cellular metabolism and overall organism functioning, including transport of oxygen, energy production and cell proliferation [15]. Patients suffering from iron deficiency may complain about fatigue, tiredness, decreased concentration and eventually anemia. Iron deficiency without anemia may be manifested by weakness, sleep disorders, restless legs syndrome, attention deficit or infertility in women [16-18]. In patients with IBD, iron deficiency anemia and anemia of chronic disease often overlap and knowing its etiology is crucial for correct management.

Iron deficiency may be caused by malnutrition with poor oral supply of the element, impaired iron absorption or chronic bleeding from the intestinal mucosal ulcers. The IDA diagnostic criteria vary depending on whether the patient is in remission or in the active phase of the disease. Therefore, in patients without endoscopic, biochemical and clinical features of disease exacerbation, low ferritin levels $\leq 30 \mu \mathrm{g} / \mathrm{l}$ indicate iron deficiency, while in case of exacerbation the cut-off is higher $-100 \mu \mathrm{g} / \mathrm{l}$. On the other hand, serum soluble transferrin receptor (sTfR) concentrations are independent from the inflammatory state and are indicators of overall iron metabolism. It is very important to screen for anemia knowing that parenteral iron supplementation seems to increase the patient's quality of life in the case of both anemic and non-anemic iron deficiency.

\section{Anemia of chronic diseases}

Anemia of chronic diseases is associated with and caused by a chronic inflammatory state. In case of active inflammation, there is an increase in the production of cytokines that stimulate the production of hepcidin in the liver. It decreases iron exports from macrophages to the endoplasmic reticulum by reduction of ferroportin. Therefore there is a reduction of transferrin saturation and iron transport to the erythroblasts, creating functional iron deficiency (FID) for erythropoiesis [19]. In addition, high levels of hepcidin reduce the absorption of iron in the duodenum [20]. The described process can be observed in many medical conditions including IBD, cancer and rheumatic diseases. In presence of biochemical or/and clinical evidence of inflammation, ferritin levels above $100 \mu \mathrm{g} / \mathrm{l}$ and transferrin saturation below $20 \%$ are diagnostic for ACD. Normal MCV does not preclude iron deficiency.

\section{Management of iron deficiency anemia}

Treatment of anemia should not be overlooked and should be started as soon as possible, as iron replacement improves quality of life of anemic and hypoferritinemic patients with IBD [21]. The decision to initiate the therapy and route of administration depend on a number of factors including the general condition of the patient, IBD activity, anemia severity and the prior 
tolerance of oral iron preparations. According to ECCO recommendations $(2 \mathrm{E})$ oral iron preparations may be used in patients in remission and with moderate anemia and in whom intolerance for oral iron (such as nausea, diarrhea, constipation and abdominal pain) preparations was not reported. Large amounts of iron in the intestinal lumen can cause oxidative stress and inflammation of the gut, leading to IBD exacerbation. Moreover, Zimmermann et al. observed that oral iron preparations may impair the gastrointestinal microbiome and increase fecal calprotectin [22]. For that reason, the maximum daily dose of basic iron in patients with IBD should not exceed $100 \mathrm{mg}$. According to the evaluation of prospective studies conducted by Nielsen et al., parenteral formulations were more effective than oral treatments in relation to an increase in hemoglobin $\mathrm{Hb})$ levels in the case of severe anemia. In mild anemia higher ferritin without elevation of $\mathrm{Hb}$ was observed in patients who received iron i.v. [23].

Indications for parenteral iron supplementation are intolerance of oral iron preparations, severe anemia $(\mathrm{Hg}<10 \mathrm{~g} / \mathrm{l})$ and need for erythropoiesis stimulants. It is not advisable to use intramuscular preparations because of many side effects, such as painful administration.

Patients with ACD and insufficient response to iron therapy should be considered for treatment with erythropoiesis. For severe anemia, blood transfusions followed by possible iron supplementation depending on hemoglobin levels should be considered.

Intravenous iron supplementation is the preferred route of iron supplementation in patients with IBD. The following preparations are available on the Polish pharmaceutical market: iron (III)-hydroxide dextran complex (Cosmofer), iron (III) isomaltoside (ISM) (Monover), iron sucrose (Venofer) and ferric carboxymaltose (FCM) (Ferinject). For the administration of dextran, the test dose is indicated. For the remaining drugs, only a 30-minute observation of the patient is indicated. In the past, to determine the correct iron dose as above, the Ganzoni formula [23] was used: total iron deficiency [mg] = body weight $[\mathrm{kg}] \times($ target hemoglobin - actual hemoglobin $[\mathrm{g} / \mathrm{dl}]) \times 0.24+500$.

On the other hand, a simplified formula has been proposed. In the FERGIcor study, the previously used scheme and the new simplified scheme (Table II) of iron supplementation were compared. New scheme proved to be more practical and efficient than the complicated Ganzoni formula. The study was carried out for iron carboxymaltose. In the case of severe anemia ( $\mathrm{Hg} 7 \mathrm{~g} / \mathrm{l})$ an additional dose of $500 \mathrm{mg}$ i.v. iron is indicated [24].

To date, there are limited data comparing different parenteral iron preparations in the IBD population. Iron isomaltoside can be administered with a maximum single dose of $20 \mathrm{mg} / \mathrm{kg}$ actual body weight, while ferric carboxymaltose can be administered with a maximum single dose of $1000 \mathrm{mg}$. A recent meta-analysis highlights that ferric carboxymaltose provides faster correction of $\mathrm{Hb}$ and serum ferritin levels in iron-deficient patients, with good tolerability of medication [25]. Iron isomaltoside appears to be less associated with hypophosphatemia than ferric carboxymaltose.

\section{Prevention of iron deficiency anemia}

Patients should be monitored every 3 months for 1 year and then every 6-12 months after receiving treatment. In case of decrease of ferritin under 100 $\mu \mathrm{g} /$ l, i.v. iron supplementation is indicated.

\section{Treatment of anemia not caused by iron deficiency}

The causes of anemia in IBD patients may vary, e.g. $A C D$, folic acid and vitamin $B_{12}$ deficiency, anemia induced by immunosuppressants or bone marrow aplasia. Their treatment depends on the cause of anemia.

\section{Hypophosphatemia related to parenteral iron infusion}

Parenteral iron preparations are considered as safe but still may lead to hypophosphatemia and hypersensitivity reactions. Iron-induced hypophosphatemia was reported for the first time in 1982 as a complication of repeated i.v. therapy with saccharated iron oxide [26]. According to the 2008 FDA report, $2.1 \%$ of patients treated with intravenous carboxymaltose developed hypophosphatemia. Hardy and Vandemergel reported a high prevalence of severe hypophosphatemia (32.1\%) after treatment of iron deficiency with high-dose intravenous ferric carboxymaltose in patients with gastrointestinal disorders [27]. According to the study of Bager et al. which compared the effects of ferric carboxymaltose and iron isomaltoside, hypophosphatemia occurred more often in patients who received FCM than the latter drug (up to $50 \%$ vs less than $10 \%$ of patients), which confirmed the previous data [28].

Phosphate plays a crucial role in homeostasis and takes part in many biological processes. It is a key mol-

Table II. Dosage of iron according to patient's weight

\begin{tabular}{lcc}
$\begin{array}{l}\text { Hemoglobin } \\
{[\mathrm{g} / \mathrm{dl}]}\end{array}$ & $\begin{array}{l}\text { Body weight } \\
<70 \mathrm{~kg}[\mathrm{mg}]\end{array}$ & $\begin{array}{c}\text { Body weight } \\
>70 \mathrm{~kg}[\mathrm{mg}]\end{array}$ \\
\hline $\begin{array}{lcc}10-12 \text { women } \\
10-13 \text { men }\end{array}$ & 1000 & 1500 \\
\hline $7-10$ & 1500 & 2000
\end{tabular}


ecule for nucleic acid synthesis, intracellular signaling and bone formation. In healthy subjects, phosphate is obtained primarily from the diet and its metabolism is regulated by factors such as parathyroid hormone and vitamin D. The mechanism of hypophosphatemia occurring after the administration of different parenteral iron is not yet fully understood, but it is probably related to increased secretion of fibroblast growth factor 23 (FGF23). The peptide binds the FGF receptor on target cells along with such cofactors as the protein Klotho and downregulates proximal renal tubule phosphate co-transporters. It induces phosphaturia and suppresses levels of 1,25-dihydroxyvitamin D. Recent data suggest that iron deficiency may stimulate FGF23 transcription in osteocytes [29]. According to a study of Wolf et al. on the effect of a single dose of FCM or iron dextran on intact, active hormone FGF23 (iFGF23) and biologically inactive, C-terminal FGF23 (cFGF23) levels in iron-deficient, anemic women, iron deficiency anemia was associated with markedly increased cFGF23 levels and normal iFGF23. Moreover, ferric carboxymaltose caused an increase of iFGF levels and subsequently decreased serum phosphate, 1,25-dihydroxyvitamin D and calcium levels, followed by the increase of PTH. This cascade was accentuated in those individuals who experienced the greatest phosphate decrease.

The lowest HP occurs 1-2 weeks after iron administration [30] and the median time of normalization after a single iron infusion is 84 days, but may be prolonged after repetitive i.v. iron infusions.

There are known cases of symptomatic hypophosphatemia following intravenous iron administration such as fatigue or muscle weakness, and even osteomalacia or secondary bone fractures for long-term parenteral iron supplementation [31, 32]. Symptomatic hypophosphatemia is more commonly associated with chronic iron use, low levels of ferritin and gastrointestinal disorders and malnutrition. The management of drug-induced symptomatic hypophosphatemia involves immediate discontinuation of intravenous iron treatment, together with phosphorus oral supplementation and dietary calcium restriction. Vitamin D deficiency should be screened and supplemented in case of deficiency, as it may help to increase phosphate intestinal absorption, even though calcitriol cannot correct FGF23 excess. Calcium and phosphorus levels should also be monitored in symptomatic patients. Treatment should be applied on the day of iron administration and on a daily basis for 3-4 weeks.

\section{Conclusions}

The most common cause of anemia in IBD is multifactorial - iron deficiency, chronic anemia, drug ef- fects or deficiency of vitamin $B_{12}$ and folic acid. It is important to screen and treat it accordingly. Intravenous preparations of iron are capable of delivering a high dosage of iron in a single or low number of visits, increasing the patient's quality of life. Even though i.v. iron has minimal potential effects, physicians should be aware of side effects which they may cause such as hypophosphatemia. Frequent check-ups should be carried out.

\section{Conflict of interest}

The authors declare no conflict of interest.

\section{References}

1. Ott C, Schölmerich J. Extraintestinal manifestations and complications in IBD. Nat Rev Gastroenterol Hepatol 2013; 10 : 585-95.

2. Kulnigg S, Gasche C. Systematic review: managing anaemia in Crohn's disease. Aliment Pharmacol Ther 2006; 24: 1507-23.

3. Wilson A, Reyes E, Ofman J. Prevalence and outcomes of anaemia in inflammatory bowel disease: a systematic review of the literature. Am J Med 2004; 116 Suppl 7A: 44S-9S.

4. Burisch J, Vegh Z, Katsanos KH, et al. Occurrence of anaemia in the first year of inflammatory bowel disease in a European population-based Inception Cohort - An ECCO-EpiCom Study. J Crohns Colitis 2017; 11: 1213-22.

5. Wells CW, Lewis S, Barton JR, et al. Effects of changes in hemoglobin level on quality of life and cognitive function in inflammatory bowel disease patients. Inflamm Bowel Dis 2006; 12: $123-30$

6. Pizzi LT, Weston CM, Goldfarb NI, et al. Impact of chronic conditions on quality of life in patients with inflammatory bowel disease. Inflamm Bowel Dis 2006; 12: 47-52.

7. Wells CW, Lewis S, Barton JR, et al. Effects of changes in hemoglobin level on quality of life and cognitive function in inflammatory bowel disease patients. Inflamm Bowel Dis 2006; 12: $123-30$.

8. Haemoglobin concentrations for the diagnosis of anaemia and assessment of severity WHO/NMH/NHD/MNM/11.1.

9. Stein J, Hartman F, Dignass AU. Diagnosis and management of iron deficiency anemia in patients with IBD. Nat Rev Gastroenterol Hepatol 2010; 7: 599-610.

10. Wiskin AE, Fleming BJ, Wootton SA, et al. Anaemia and iron deficiency in children with inflammatory bowel disease. J Crohns Colitis 2012; 6: 687-91

11. Goodhand JR, Kamperidis N, Rao A, et al. Prevalence and pathogenesis of anemia in inflammatory bowel disease. Influence of anti-tumor necrosis factor-alpha treatment. Haematologica 2010; 95: 199-205.

12. Wilkholm E, Malmborg P, Forssberg M, et al. Iron deficiency is common during remission in children with inflammatory bowel disease. Glob Pediatr Health 2016; 3: 2333794X16633672.

13. Dignass AU, Gasche C, Bettenworth D, et al. European Consensus on the diagnosis and management of iron deficiency and anaemia in inflammatory bowel diseases. J Crohns Colitis 2015; 9: 211-22. 
14. Kaniewska M, Bartnik W, Gonciarz M, et al. Iron deficiency anaemia in patients with inflammatory bowel disease: National Consultant for Gastroenterology Working Group Recommendations. Prz Gastroenterol 2014; 9: 259-63.

15. Hentze MW, Muckenthaler MU, Galy B, et al. Two to tango: regulation of mammalian iron metabolism. Cell 2010; 142: 24-38.

16. Brunner AV, Joffe A, Duggan AK, et al. Randomised study of cognitive effects of iron supplementation in non-anemic iron-deficient adolescent girls. Lancet 1996; 348: 992-6.

17. Chavarro JE, Rich-Edwards JW, Rosner BA, et al. Iron intake and risk of ovulatory infertility. Obstet Gynecol 2006; 108: 1145-52.

18. KrayenbuehI PA, Battegay E, Breymann C, et al. Intravenous iron for the treatment of fatigue in nonanemic, premenopausal women with low serum ferritin concentration. Blood 2011 118: 3222-7.

19. Weiss G, Goodnough LT. Anemia of chronic disease. N Engl J Med 2005; 352: 1011-23.

20. Bregman DB, Morris D, Koch TA, et al. Hepcidin levels predicts nonresponsiveness to oral. Am J Hematol 2013; 88: 97-101.

21. Elena E, Gregory K, Judy $\mathrm{H}$, et al. Intravenous iron replacement improves quality of life in hypoferritinemic inflammatory bowel disease patients with and without anemia. Dig Dis 2017; 35: 444-8.

22. Zimmermann MB, Chassard J, Rohner F, et al. The effects of iron fortification on the gut microbiota in African children: randomized control trial in Cote d'Ivoire. Am J Clin Nutr 2010; 92: 1406-15.

23. Nielsen O, Ainsworth M, Coskun M, et al. Management of iron-deficiency anemia in inflammatory bowel disease: a systemic review. Medicine 2015; 94: e963.

24. Ganzoni AM. Intravenous iron-dextran: therapeutic and experimental possibilities. Schweiz Med Wochenschr 1970; 100: 301-3.

25. Evstatiev R, Alexeeva O, Bokemeyer B, et al. Ferric carboxymaltose prevents recurrence of anemia in patients with inflammatory bowel disease. Clin Gastroenterol Hepatol 2013; 11: 269-77.

26. Rognoni C, Venturini S, Meregaglia $M$, et al. Efficacy and safety of ferric carboxymaltose and other formulations in iron-deficient patients: a systemic review and network meta-analysis of randomised controlled trials. Clin Drug Investig 2016; 36 177-94.

27. Hardy S, Vandemergel X. Intravenous iron administration and hypophosphatemia in clinical practice. Int J Rheumatol 2015; 2015: 468675

28. Bager P, Hvas C, Dahlerup J. Drug-specific hypophosphatemia and hypersensitivity reactions following different intravenous iron infusions. Br J Clin Pharmacol 2017; 83: 1118-25.

29. Farrow EG, Yu X, Summers LJ, et al. Iron deficiency drives an autosomal dominant hypophosphatemic rickets (ADHR) phenotype in fibroblast growth factor-23 (Fgf23) knock-in mice. Proc Natl Acad Sci U S A 2011; 108: E1146-55.

30. Okada M, Imamura K, Fuchigami T, et al. Two cases of nonspecific multiple ulcers of the small intestine associated with osteomalacia caused by long-term intravenous administration of saccharated ferric oxide. Nihon Naika Gekkai Zasshi 1982; 71: 1566-72.
31. Schaefer B, Würtinger P, Finkenstedt A, et al. Choice of highdose intravenous iron preparation determines hypophosphatemia risk. PLoS One 2016; 11: e0167146.

32. Bishay R, Granada K, Seibel M. Long-term iron polymaltose infusions associated with hypophosphataemic osteomalacia: a report of two cases and review of the literature. Ther Adv Endocrinol Metab 2017; 8: 14-9.

Received: 1.12 .2017

Accepted: 4.12 .2017 